Journal of Computer Science 8 (6): 971-977, 2012

ISSN 1549-3636

(C) 2012 Science Publications

\title{
Congestion Free Routing in Adhoc Networks
}

\author{
${ }^{1}$ Senthil Kumaran, T. and ${ }^{2}$ V. Sankaranarayanan \\ ${ }^{1}$ Department of CSE, \\ BSA Crescent Engineering College, \\ ${ }^{2}$ B.S.Abdur Rahman University Vandalur, \\ Chennai, Tamilnadu, India
}

\begin{abstract}
Problem statement: A Mobile Ad-Hoc Network (MANET) is a temporary network; the mobile devices in an ad-hoc network are communicating through wireless links without any pre-existing infrastructure. The one major problem of this network is network congestion, it may take place at any intermediate nodes when data packets are traveling from source to destination. The major problems of congestion or high data loss, increasing End to End and retransmission packets which affects the overall network performance. The main goal of congestion control is to effectively utilize the existing network resources and maintain the network load below the capacity. Approach: This study presents the Congestion Free Routing in Adhoc networks (CFR), based on dynamically estimated mechanism to monitor network congestion by calculating the average queue length at the node level. While using the average queue length, the nodes' congestion status divided into the three zones (safe zone, likely to be congested zone and congested zone). CFR utilizes the non-congested neighbors and initiates route discovery mechanism to discover a congestion free route between source and destination. This path becomes a core path between source and destination. To maintain the congestion free status, the nodes which are helping data packet transmission periodically calculate their congestion status at the node level. When a core node is noticed that it fell in to likely to congested zone and alerts to its neighbors. The predecessor core path node is aware of this situation and initiates an alternate path discovery mechanism to a destination. Finally it discovers a new congestion free route to the destination. Results: The assessment between CFR and AODV was conducted by using the Ns-2 simulator. The simulation results confirmed that the CFR improved packet delivery ratio, reduction of End to End delay and control packets. Conclusion: Our proposed technique solved the congestion problems in a network.
\end{abstract}

Key words: Mobile Ad Hoc Networks (MANETs), Random Early Detection (RED), Active-QueueManagement (AQM), Congestion Status Packet (CSP), Congestion Free Routing in Adhoc networks (CFR), reliable communication

\section{INTRODUCTION}

In recent times, a number of research techniques and applications have been used widely for transmitting information through heterogeneous wireless networks. The wireless network can be characteristics into a local area wireless network, ad hoc network and Satellite wireless network. Mobile Ad hoc Network (MANET) is one kind of wireless network that does not need a backbone infrastructure to be set up for communication and data delivery. The advantages of adhoc network is Ease of deployment than wired networks, Scalability and Flexibility (Murthy and Manoj, 2004; Ramanathan and Redi, 2002; Yen et al., 2010).

Congestion in a network may occur at interval time when the incoming traffic is larger than the capacity of the network. This network congestion can severely increase delay and packet loss and cut the network throughput. Congestion control refers to techniques that can keep away from congestion before it happens or recovery after it happens (Lochert et al., 2007; Kumaran and Sankaranarayanan, 2011a).

The main aim of congestion control is to lower the EndtoEnd delay and reduced packet lost caused by network congestion and offer better performance of the network (Lochert et al., 2007; Tran and Raghavendra, 2006; Kumaran and Sankaranarayanan, 2011b). In wire line networks, congestion control is employed at the transport layer and it is independent from the functionality of other layers (Lochert et al., 2007; Tran and Raghavendra, 2006; Yu and Giannakis, 2008). However, these congestion control techniques do not apply directly to ad hoc networks, because the ad hoc network is challenged by a limited wireless bandwidth,

Corresponding Author: Senthil Kumaran, T., Department of CSE, BSA Crescent Engineering College, Chennai, Tamilnadu, India 
power constraints and route failures, due to node mobility and limited buffer size. The final result is a high packet-loss rate, re-routing instability, loss of energy, bandwidth and retransmission of lost packets, which implies that more packets are transmitted in the network. These delays and packet losses are not originated by network congestion, but this can be misinterpreted as congestion losses (Kumaran and Sankaranarayanan, 2011c).

In ad hoc networks, the routing protocols for MANETs are classified into three categories (i) proactive, (ii) reactive and (iii) hybrid (joined both proactive and reactive). The Examples of proactive routing protocols are DSDV and OLSR (Perkins and Bhagwat, 1994; Murthy and Manoj, 2004; Chen and Heinzelman, 2007). The example of reactive routing protocols is AODV (Perkins et al., 2003; Murthy and Manoj, 2004) and DSR. The hybrid routing protocol is ZRP (Murthy and Manoj, 2004; Chen and Heinzelman, 2007).

There is a new aspect to categorize routing protocols into two divisions (i) congestion-control routing and (ii) congestion non control routing. (Lochert et al., 2007; Tran and Raghavendra, 2006).

When we consider the congestion non control routing protocol, during the packet transfer between the source and destination, congestion may occur; this is not managed by the existing routing protocol.

The above problems turn into the harmful in a network in terms of packet loss, increasing delay and reduced throughput. (Lochert et al., 2007; Tran and Raghavendra, 2006). The exsisting congestion control techniques cannot directly used in an adhoc network because in an adhoc network, it is more expensive, in terms of time and overhead and removes congestion after it happened (Lochert et al., 2007; Tran and Raghavendra, 2006).

To relieve the network congestions, many researchers have promoted the use of Active-QueueManagement (AQM) strategies.

This primary design is that to offer preventive rules for managing a node's buffer effetivelly and cut those problems related with probable congestions (Athuraliya et al., 2001).

Many AQM techniques, such as the adaptive virtual queue, Random Early Detection (RED), random exponential marking, PI controller (Athuraliya et al., 2001) and the blue and stochastic blue (Feng et al., 2001) schemes, have been reported. Among these existing schemes, the AQM scheme is recommended by the IETF for the next generation Internet routers is the Random Early Detection (RED) (Braden et al., 1998). This is because it predicts the congestion by observeing the average queue size.
This study is to bring a novel outlook of this problem and an anticipate congestion at the MAC layer due to buffer overflow and adapt the traffic in the network layer by finding a non-congested path. This technique is necessary to avoid or cut packet loss, reduction of delay and improve the overall network performance.

In our previous study, Early Detection congestion and control routing (EDAODV) (Kumaran and Sankaranarayanan, 2010) techniques have been proposed to detect the congestion well in advance and find a non-congested alternate path bi-directionally.

A technique for self curing the congestion was proposed in (Kumaran and Sankaranarayanan, 2010) and is called the Early congestion detection and self cure routing (EDCSCAODV).

In EDOCR (Early congestion detection and optimal control routing), the network is divided in to sparse and dense regions by using average neighbors, to find a non-congested alternate path with the help of dense nodes (Kumaran and Sankaranarayanan, 2011b).

In EDAPR (Kumaran and Sankaranarayanan, 2011c) (Early congestion detection and adaptive routing) techniques have been proposed for preventing congestion by using the NHN (Non-congested 2 hop neighbors list).

our proposed CFR uses a new algorithm by detecting congestions dynamically. It uses a noncongested path discovery mechanism to prevent network congestion. Hence, congestion is resolved prior to happen, it occurrences so, it decreases data packet loss, EndtoEnd delay and improved throughput.

\section{MATERIALS AND METHODS}

Dynamic Congestion estimation technique (DC): Congestion may happen at any point of time in a network then node becomes congested and starts losing packets. In an Adhoc network, we used node level congestion mechanism to measure the packet loss due to lack of buffer space . Every second a node checks the residence of packet in its queue by applying dynamic congestion estimation technique. The DC algorithm utilizes three parameters like the Minth, Maxth and Wq.

The Eq. 1 and 2 are helped to assign the Minimum threshold and Maximum threshold values. The fixing of the Min threshold and Max threshold value depend on preferred average queue size. In our algorithm, we chose to fix the minimum threshold of $35 \%$ (Floyd and Jacobson, 1993; Floyd, 1993).

$$
\begin{aligned}
& \text { Minth }=35 \% \text { Queue_size } \\
& \text { Maxth }=2 * \text { Minth }
\end{aligned}
$$


The purpose of the average queue length is to join all the traffic fluctuations and it chases the continuing changes of the Instant queue, imitating the constant congestion in networks. Equation 3 supported to find an average queue length:

$$
\text { Avgque }=\left(1-\mathrm{w}_{\mathrm{q}}\right) * \text { Avgque }+ \text { Inst_Que } * \mathrm{w}_{\mathrm{q}}
$$

The weight factor $\mathrm{Wq}$ regulates the network congestion. If the $\mathrm{Wq}$ is too tiny, the average length of the queue does not clutch the extensive range congestion, which might result in ineffective Congestion Detection Technique. If $\mathrm{wq}$ is an outsized, the average queue length follows the instant queue, which corrupts the performance of the Congestion Estimation Technique. Therefore, the value of $\mathrm{Wq}$ should be communicated to the traffic smoothing in the queue.

The proposed Dynamic congestion estimation technique would concentrate on assigning $\mathrm{Wq}$ values dynamically according to the traffic flow. Initially, the Wq is set to 0.002 (Floyd and Jacobson, 1993; Floyd, 1993). The Eq. 4 is used to set Wq values dynamically, where $\mathrm{N}$ is the some lively flows and $\mathrm{P}$ is the packet rate (no: of packets per second):

$$
\mathrm{Wq}=\mathrm{Wq} * \mathrm{~N} * \mathrm{P}
$$

If the Avgque length is smaller than the Minth and instant queue < warn_line (warn_line = queue_size/2), then the node's congestion status becomes Zone I (safe zone). If the Avgque is larger than Minth and samller than Maxth, then the node's congestion status becomes Zone-II (likely to be a congested zone) and initiates an alternative path discovery mechanism. Finally, if the Aveque is bigger than Maxth, then the node's congestion status becomes Zone -III (congested zone).

Congestion free route discovery: Each mobile node chooses its CFS (Wu et al., 2006; Yen et al., 2010) set from its non-congested 1-hop neighbors. The CFS is chosen in such a method that it wraps all 2-hop nodes.

The CFS set of source host S, represented by CFS(S), then a random subset of the non-congested 1hop region of $\mathrm{S}$ which convinces condition: Every node in the exacting 2-hops zone of $\mathrm{S}$ must have a link towards CFS(S) and it should not fall in the congested zone. The CFS setup is an initialization procedure, where each mobile host every second calculates its congestion status by using the dynamic congestion estimation technique. Every mobile host broadcasts its congestion status by using a Congestion Status Packet (CSP) to its one hop neighbors on the network. Now, each mobile node discovers about its 1-hop non- congested neighbor nodes and accumulates the information about its 1-congested one-hop list. At this point, each mobile node builds its CFS-set by selecting a subset of its 1-hop non-congested neighbor nodes, so that the mobile node in the subset can send its broadcast packet to the 2-hop neighbor nodes, to decrease the overflow traffic. Each mobile node updates all the information in its routing table.

When the source node desires to communicate a data packet to a destination, the source node creates the RREQ packet for broadcasting using the CFS-set nodes towards the destination. The source node initially verifies its 2-hop list. If the destination host is present with in 2-hop list, then the RREQ is transmitted by after the routing table's path. If the destination node is not with in 2-hop list, the source host broadcasts the RREQ to the CFS-set in a network.

When the CFS-set obtains RREQ packet and checks its 2-hop list. If the destination is with in its 2hop list, then the CFS node delivered the RREQ to the destination node. The destination answered to the first received RREQ and replied an RREP packet to the source node and add a new entry in its routingtable.

Figure 1 shows the route discovery subsequent to the CFS-set selection. The source node $\mathrm{S}$ has a noncongested 1-hop lists are $\{2,3,4\}$ and a non-congested 2-hop lists are $\{4,6,7\}$. The source has chosen node 3 as a CFS and added it to the CFS list. The first node $\mathrm{S}$ verifies its 2-hop list to check whether it contains the destination node $\mathrm{D}$. If the destination node $\mathrm{D}$ is not with in the list, the source node $\mathrm{S}$ broadcast the RREQ packet to the next CFS node 3. Then, node 3 would verifies the 2-hop list. If the destination is not inside, the CFS node 3 broadcast the RREQ to the next CFS node 6 ; The CFS node 6 would verifies the 2-hop list. If the destination is not present, then the CFS node 6 broadcasts RREQ to the next CFS node 9; now node 9 discovers the destination node $\mathrm{D}$ is in the 2-hop list; so node 9 forwards RREQ packet by CFS node 11 to the destination node D. Destination node D gets the RREQ packet and then replies the RREP packet to the source. A route $S \rightarrow 3->6>9->11>D$ is found between source $S$ and destination $\mathrm{D}$. This path becomes non-congested path between the source and the destination. After the route discovery, the data packet is sent between source and destination. This route became the core route from $\mathrm{S}$ to $\mathrm{D}$.

Congestion free alternative path discovery: A core path of a nodes predict their congestion status periodically and updates their congestion status by broadcasting CSP packet with TTL $=1$. When the precursor node receives a CSP packet from its core path node of say A regarding destination $\mathrm{D}$, precursor node will be alert of the congestion information of A, Non congested node in the core path and its hop count. 


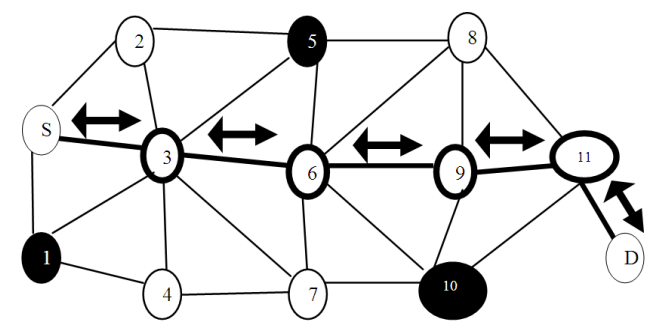

Fig. 1: Route discovery process through CFS set $\leftrightarrow$ Route Request, Replay

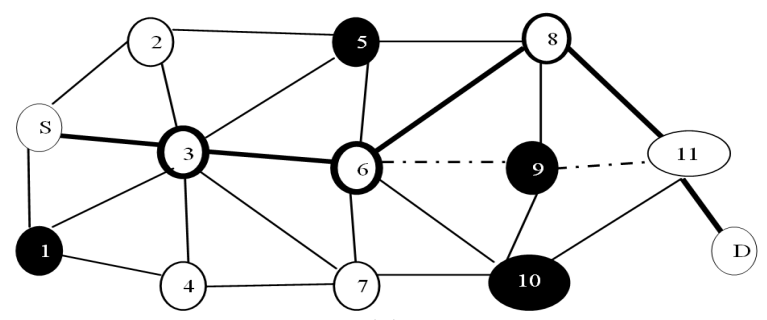

(a)

Fig. 2: Alternate path finding process Congested node $\cdots \cdot$ primary path - Alternate path

When the descendant node receives a CSP packet from its core path node of A regarding to the Source $\mathrm{S}$, descendant node will be awake of the congestion information of A, previous Non-congested node in the core path and its hop count. The routing table of ancestor and an heir nodes are keep informed accordingly. This information is step forward to find the bidirectional non-congested alternate path:

$\begin{array}{ll}\text { Routing } & \text { CFR, AODV } \\ \text { MAC } & 802.11 \\ \text { Bandwidth } & 2 \mathrm{Mbps} \\ \text { Terrain } & 1400,1400 \mathrm{~m} \\ \text { Nodes } & 100 \\ \text { Antenna } & 2 \text { ray ground } \\ \text { Node placement } & \text { Uniform } \\ \text { Data traffic } & \text { CBR } \\ \text { Simulation time } & 900 \mathrm{sec} \\ \text { MAC queue size } & 50 \text { packets } \\ \text { Routing queue } & 54 \text { packets } \\ \text { Load (Flows) } & 10-50 \mathrm{Flows} \\ \text { Load (Pkts/Seconds) } & 4-16 \mathrm{Pkts} / \mathrm{S} \\ \text { Max Sped (m/s) } & 0-10 \mathrm{~m} \mathrm{sec}^{-1} \\ \text { Pause Time(s) } & 30 \mathrm{sec}\end{array}$

Concurrently, an heir node (B) receives a CSP packet from its prior core node $P_{-}$Node (about Source $\mathrm{S})$. If (A) is a non -congested node (ZoneI node), (A) starts to discover an alternate path towards node
N_ZoneI - [the next non-congested node of (A) known from the CSP packet]. Similarly if (B) is a noncongested node (ZoneI node), (B) starts to discover an alternate path towards node P_ZoneI - [the previous noncongested node of (B) known from the CSP packet]. Suppose (A) is not a non-congested node (ZoneI node) it sends CSP packet to its predecessors until it reaches noncongested node (ZoneI node) and if (B) not, (B) does the same procedure until CSP reaches successor noncongested node (ZoneI node). Finally, CFR finds primary path non-congested nodes on both sides (Predecessor P_ZoneI, successor N_zoneI). As a result, the secondary path should be disjoint with the core path, excluding that they link at the end nodes P_ZoneI and N_ZoneI. It is probable that no secondary path is found, In this case, all the core path nodes are continue using the core route. Our secondary path is more elastic and not essentially the shortest path therefore, the cost of finding secondary path is even higher.

Figure 2 shows how the CFS node 9 notices that congestion is likely to occur and sends a warning to its neighbors CFS node 6 and 11; they are aware of this situation and update their non-congested neighbor list in their routing table. In response, the processor CFS node 6 chooses a new CFS node 8 from its non-congested neighbor list, because node 8 is a common node for node 6 and node 8 and it finds the route to the destination using CFS node 8 as shown in Fig. 2. The traffic coming to 6 will be routed through the new route $\mathrm{s}->3->6$->8 $>11$-> D respectively. It is possible that if no CFS nodes are found, it continues using the primary route $S$-> 3 -> 6 $\rightarrow>9->11>\mathrm{D}$. The new path is a non-congested path, but not necessarily the shortest path.

Performance metrics: The CFR and AODV are implemented using the Network Simulator (NS). A comparison of the CFR's performance with that of the AODV routing protocols is made in MANET:

- Packet Delivery Ratio (PDR): The ratio between received packet and sent packet.

- End-to-End Delay: The delay a packet suffers from the source to the receiver.

- Routing overhead: The total number of RREQ,RREP,RERR and CSP packets transmitted during the simulation time.

\section{RESULTS AND DISCUSSION}

Varying number of connections: In this simulation, the number of sources and destinations are varied from 1050, CBR interval 8 packets per second, maximum node speed $10 \mathrm{~m} \mathrm{sec}^{-1}$ and pause time $30 \mathrm{sec}$. Figure 3a-c have shown the End-to-End delay, packet delivery ratio and Routing overhead for CFR and AODV respectively. 


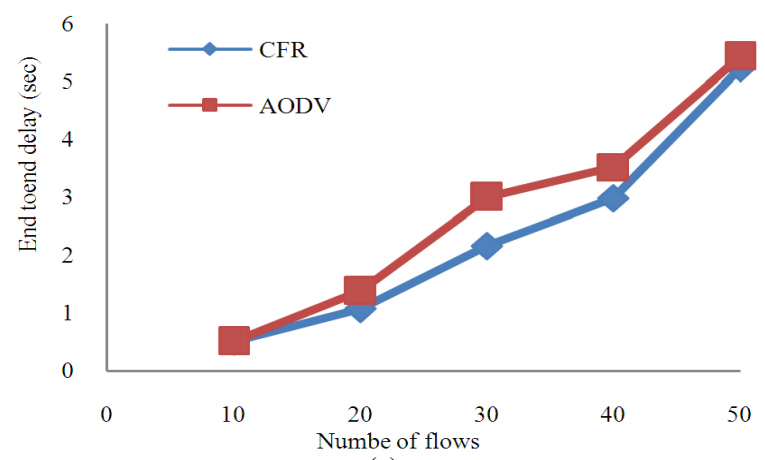

(a)

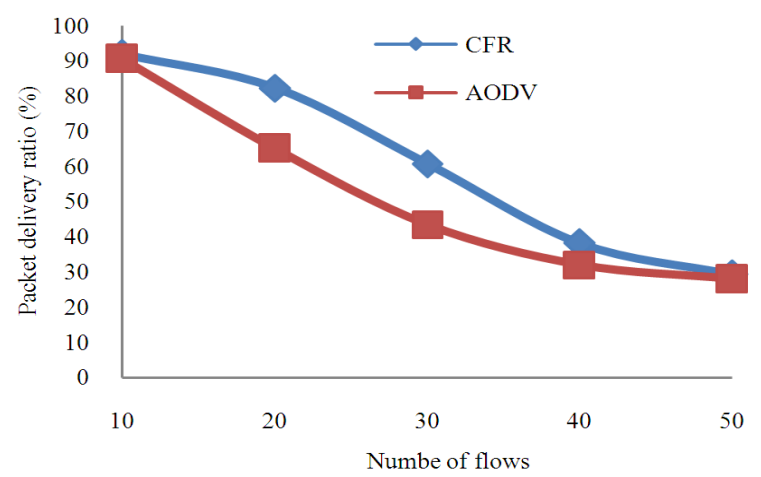

(b)

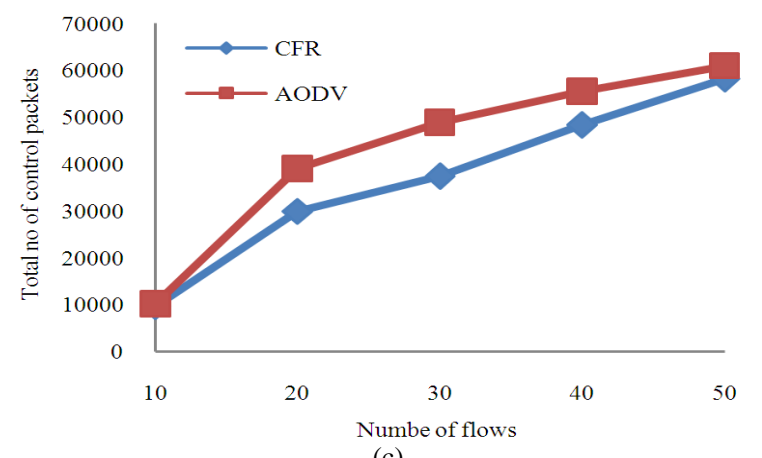

(c)

Fig. 3: Performance, when number of connections (source and destination) change (a) End to End delay (b) Packet delivery ratio (c) Routing overhead

The results in Fig. 3a show that the delays acquired by the two protocols (CFR and AODV) are similar when the number of flows set at 10 . This is because at a low offered load, the network becomes a safe zone. In the case of a high offered load (between 20 and 30 flows), the network congestion status becomes a likelyto-congestion zone; the CFR routing protocols demonstrate around $28 \%$ reduction in delay over the AODV. When the number of flows between 30 and 40, the CFR delay is reduced by around $15 \%$ over AODV. Figure $3 \mathrm{~b}$ shows the achieved packet delivery ratio of the two protocols, which is similar when the offered load is below 20 flows. When the no of flows increasesfrom 20-50, as an outcome, more control packets are generated and conveyed, this leads to a high utilization of the node's queue, causing network congestion. This, in fact, leads to a smaller amount of data packets being delivered to the destinations, The net result degrading the overall network's performance. But, it can be noticed from Fig. 3b that initially, the CFR constructed a 2 hops CFS set. It knows all the noncongested neighbors, both one hop and 2 hops neighbors, so that it takes the minimum number of control packets to find an alternate path than the AODV. At an offered load of 20-30 flows, the packet delivery ratio is increased from 20-28\% when compared with the AODV.

With regard to the routing overhead, Fig. 3c shows, that when the offered load is low (e.g., 10 flows) the CFR did not give a better performance than the AODV. This is because at a small traffic, the network became a safe zone. When the traffic is increased from 20-30 flows, the AODV incurred a heavy routing overhead and consumed the heaviest control packets to find a new path, whereas the CFR required the least control packers of around 23\%, of the overhead of the AODV. the routing overhead of the CFR being less than the AODV. When the number of flows increased from 30-40, the traffic was heavier, but the routing overhead difference seems un fair between the CFR and the AODV.

Varying the CBR load: In this simulation, the number of connections (different sources and different destinations) is reserved at 20 . The CBR sources send data packets to the destinations at different rates, varying from 4 packets/s to 40 packets/sec. One observes that the End-to-End delay in the CFR and AODV is as shown in Fig. 4a. When the data packetinterval was low (less than 8 packets $\sec ^{-1}$ ), the delay earned by the two protocols increases almost same, with increased traffic and the delay variation between the CFR and the AODV seems unchanged. Compared with the AODV at a high interval (8-16 packets $\mathrm{sec}^{-1}$ ), the delay is reduced from $22-26 \%$ over the AODV respectively. The CFR and the AODV met to a related performance when the rate was too high (30-40 packets $\mathrm{sec}^{-1}$ ) because the network gained the heaviest traffic. With regard to the packet delivery ratio Fig. $4 \mathrm{~b}$, when the packet rate was small (less than 8 packet $\mathrm{sce}^{-1}$ ), the CFR and the AODV carried similar loads of packets, because the network traffic was not yet heavy. But, when the packet rate was high (8-16 packets $\left.\sec ^{-1}\right)$, the network becomes likely to be congested, the CFR applied a CFS set, so that it finds an alternate path immediately; the CFR seems an improved at least by 21-26\% packet delivery ratio than the AODV. 


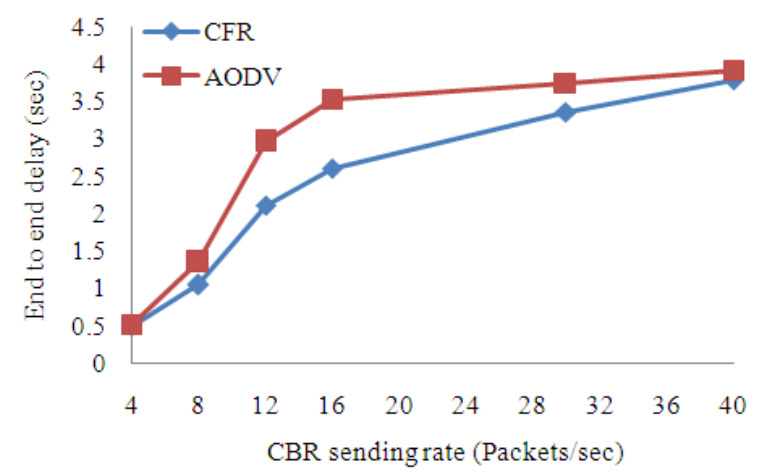

(a)

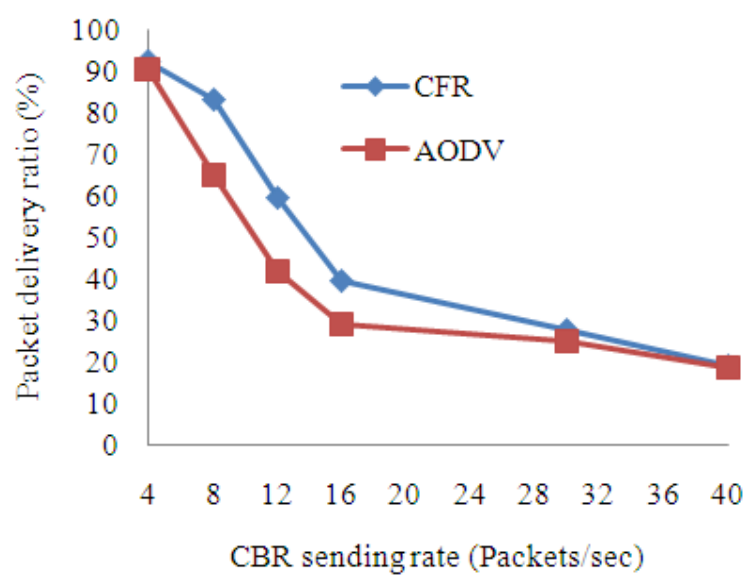

(b)

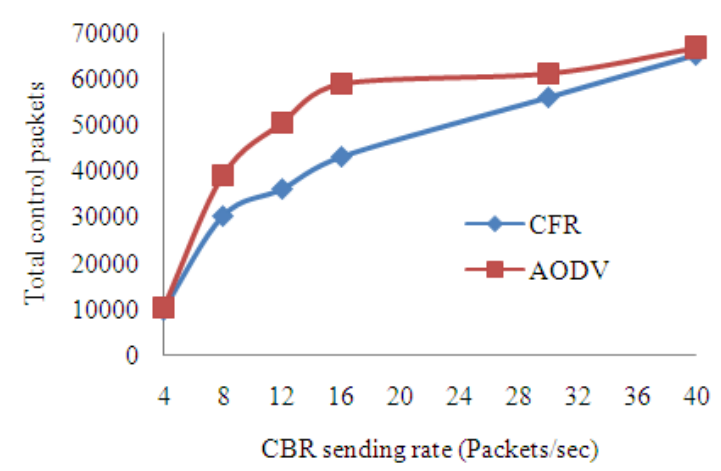

(c)

Fig. 4: Performance when the CBR load changes (a) End to End delay (b) Packet delivery ratio (c) Total Control Packets

Figure $4 \mathrm{c}$ shows the routing overhead of the CFR and the AODV. When the traffic consignment was small (4-8 packet $\mathrm{sec}^{-1}$ ), the routing overhead of the CFR and the AODV was similar. More exultingly, when the traffic was heavier (8-16 packets $\left.\sec ^{-1}\right)$, the routing overhead of the CFR was reduced from $22-26 \%$ than the routing overhead of the AODV. when more packets were produced into the network (30 or 40 packets $\sec ^{-1}$ ), the two protocols deserved the heaviest routing overhead in more stressful network and the reduction of the routing overhead by the CFR was only $2.5 \%$ over the AODV.

\section{CONCLUSION}

The objective of the congestion control mechanism is designed for multimedia applications in mobile ad hoc networks. These network characteristics, like congestion, route failure, need to be detected and remedied with a reliable mechanism. Our proposed technique tries to solve the congestion problem in this study. The CFR has used a novel way called the dynamic congestion estimation technique, which analyzed the traffic fluctuation and categorized the congestion status perfectly. After estimating the congestion status at the node level along a path, the CFR controls the congestion by using an alternative path. The CFR congestion control mechanism shows considerable performance improvement over the AODV. The ns- 2 results has confirmed that the CFR mechanism outperforms the AODV in terms of decreasing End to End delay, reduced routing overhead and increased packet delivery ratio.

\section{REFERENCES}

Athuraliya, S., S.H. Low, V.H. Li and Q. Yin, 2001. REM: Active queue management. IEEE Netw., 15: 48-53. DOI: $10.1109 / 65.923940$

Braden, B., D. Clark, J. Crowcroft, B. Davie and S. eering et al., 1998. Recommendations on queue management and congestion avoidance in the internet. RFC, United States.

Chen, L. and W.B. Heinzelman, 2007. A survey of routing protocols that support qos in mobile ad hoc networks. IEEE Netw., 21: 30-38. DOI: 10.1109/MNET.2007.4395108

Feng, W.C., D.D. Kandlur, D. Saha and K.G. Shin, 2001. Stochastic fair blue: A queue management algorithm for enforcing fairness. Proceedings of the 20th Annual Joint Conference of the IEEE Computer and Communications Societies, Apr. 2226, IEEE Xplore Press, Anchorage, AK., pp: 15201529. DOI: 10.1109/INFCOM.2001.916648

Floyd, S. and V. Jacobson, 1993. Random early detection gateways for congestion avoidance. IEEE/ACM Trans. Netw., 1: 397-413. DOI: 10.1109/90.251892 
Floyd, S., 1993. RED: Discussions of setting parameters.

Kumaran, T.S. and V. Sankaranarayanan, 2010. Early detection congestion and control routing in MANET. Proceedings of the 7th IEEE and IFIP International Conference on wireless and Optical communications Networks (WOCN), Sept. 6-8, IEEE Xplore Press, Chennai, India, pp: 1-5. DOI: 10.1109/WOCN.2010.5587363

Kumaran, T.S. and V. Sankaranarayanan, 2011a. Early congestion detection and optimal control routing in MANET. Eur. J. Sci. Res., 63: 15-31.

Kumaran, T.S. and V. Sankaranarayanan, 2011b. Early congestion detection and adaptive routing in MANET. Egyptian Inform. J., 12: 165-175. DOI: 10.1016/j.eij.2011.09.001

Kumaran, T.S. and V. Sankaranarayanan, 2011c. Early congestion detection and self cure routing in MANET. Commun. Comput. Inform. Sci., 142: 562-567.

Lochert, C., B. Scheuermann and M. Mauve, 2007. A survey on congestion control for mobile ad hoc networks: Research articles. J. Wireless Commun. Mob. Comput., 7: 655-676. DOI: 10.1002/wcm.v7:5

Murthy, C.S.R. and B.S. Manoj, 2004. Ad Hoc Wireless Networks: Architectures And Protocols. 1st Edn., Pearson Education India, India, ISBN: 8131706885, pp: 878.

Perkins, C.E., E.M. Belding-Royer and I.D. Chakeres, 2003. Ad-hoc on-demand distance vector routing. IETF Internet draft, University of California, Santa Barbara.
Perkins, C.E. and P. Bhagwat, 1994. Highly Dynamic Destination-Sequenced Distance-Vector Routing (DSDV) for mobile computers. Roceedings of the Conference on Communications Architectures, Protocols and Applications, Aug. 31-Sept. 02, ACM, London, England UK., pp: 234-244. DOI: 10.1145/190314.190336

Ramanathan, R. and J. Redi, 2002. A brief overview of ad hoc networks: Challenges and directions. IEEE Commun. Mag., 40: 20-22. DOI: 10.1109/MCOM.2002.1006968

Tran, D.A. and H. Raghavendra, 2006. Congestion adaptive routing in mobile ad hoc networks. IEEE Trans. Parallel Distribut. Syst., 17: 1294-1305. DOI: 10.1109/TPDS.2006.151

Wu, J., W. Lou and F. Dai, 2006. Extended multipoint relays to determine connected dominating sets in MANETs. IEEE Trans. Comput., 55: 334-347. DOI: $10.1109 /$ TC.2006.40

Yen, Y.S., H.C. Chang, R.S. Chang and H.C. Chao, 2010. Routing with adaptivepath and limitedflooding for mobile ad hoc networks. Comput. Elect. Eng., 36: 280-290. DOI: 10.1016/j.compeleceng.2009.03.002

$\mathrm{Yu}$, Y. and G.B. Giannakis, 2008. Cross-layer congestion and contention control for wireless ad hoc networks. IEEE Trans. Wireless Commun., 7: 37-42. DOI: 10.1109/TWC.2008.060514 